\title{
Dental Emergency Situations
}

\author{
Siniša Franjić \\ Independent Researcher, Croatia.
}

How to cite this paper: Siniša Franjić. (2020) Dental Emergency Situations. International Journal of Clinical and Experimental Medicine Research, 4(3), 63-68.

DOI: $10.26855 /$ ijcemr.2020.07.006

Received: May 18, 2020

Accepted: June 17, 2020

Published: July 1, 2020

*Corresponding author: Siniša Franjić, Independent Researcher, Croatia.

Email: sinisa.franjic@gmail.com

\begin{abstract}
Emergency conditions are situations in which the lives of patients are endangered in whom therapy should be given immediately upon the onset of symptoms. The majority of emergency conditions in dental practice can be controlled by usual therapeutic procedures, and such conditions do not disturb the vital functions of the organism for a shorter period. Every dental professional must be familiar with the possible complications that can occur in the dental practice, as well as their management.
\end{abstract}

\section{Keywords}

Emergency, Dental Emergency, Dental Pain, Treatment

\section{Introduction}

An emergency is commonly defined as any condition perceived by the prudent layperson—or someone on his or her behalf-as requiring immediate medical or surgical evaluation and treatment [1]. On the basis of this definition, the American College of Emergency Physicians states that the practice of emergency medicine has the primary mission of evaluating, managing, and providing treatment to these patients with unexpected injury and illness.

So what does an emergency physician (EP) do? He or she routinely provides care and makes medical treatment decisions based on real-time evaluation of a patient's history, physical findings, and many diagnostic studies, including multiple imaging modalities, laboratory tests, and electrocardiograms. The EP needs an amalgam of skills to treat a wide variety of injuries and illnesses, ranging from the diagnosis of an upper respiratory infection or dermatologic condition to resuscitation and stabilization of the multiple trauma patients. Furthermore, these physicians must be able to practice emergency medicine on patients of all ages. It has been said that EPs are masters and mistresses of negotiation, creativity, and disposition. Clinical emergency medicine may be practiced in emergency departments (EDs), both rural and urban; urgent care clinics; and other settings such as at mass gathering incidents, through emergency medical services (EMS), and in hazardous material and bioterrorism situations.

In healthcare delivery, we attempt to meet the health and medical needs of the community by providing a place for individuals to seek preventative medicine, care for chronic medical conditions, emergency medical treatment, and rehabilitation from injury or illness [2]. While a healthcare institution serves the community, this responsibility occurs at the level of the individual. Each individual expects a thorough assessment and treatment if needed, regardless of the needs of others. This approach is different than that practiced by emergency managers, whose goal is to assist the largest number of people with the limited resources that are available. As such, emergency management principles are focused on the needs of the population rather than the individual. When either planning for a disaster or operating in a disaster response mode, the hospital should be prepared at some point to change its focus from the individual to the community it serves and to begin weighing the needs of any individual patient versus the most good for the most patients with scarce resources. Moving from the notion of doing the most for each individual to doing the best for the many is a critical shift in thinking for healthcare institutions 
considering a program of comprehensive emergency management. While the initial planning for emergencies by hospitals is focused on maintaining operations and handling the care needs of actual or potential increased numbers of patients and/or different presentations of illness or injury than is traditionally seen, there is also the need to recognize that at some point during a disaster, act of terrorism, or public health emergency there may be an imbalance of need versus available resources. At this point, the approach to delivering healthcare will need to switch from a focus on the individual to a focus on the population. This paradigm shift is one of the core unique aspects of hospital emergency management that allows the hospital to prepare to maximize resources in disasters and then to know when to switch to a pure disaster mode of utilizing its limited and often scant resources to help the most people with the greatest chance of survival.

The healthcare delivery system is vast and comprised of multiple entry points at primary care providers, clinics, urgent care centers, hospitals, rehabilitation facilities, and long-term care facilities. The point of entry for many individuals into the acute healthcare system is through the emergency department (ED). Since the late 1970s, the emergency medical services (EMS) system has allowed victims of acute illness and injury to receive initial stabilization of life-threatening medical conditions on the way to the emergency department. Among the many strengths of the ED is the ability to integrate two major components of the healthcare system: prehospital and definitive care. The emergency department maintains constant communications with the EMS system and serves as the direct point of entry for prehospital providers into the hospital or trauma center. Emergency physicians represent a critical link in this process by anticipating the resources that ill and injured patients will need upon arrival at the ED, and initiating appropriate life-saving medical care until specialty resources become available. In this context, the healthcare system is an emergency response entity.

\section{Patient Conditions}

In most emergencies, there is no time to disclose the necessary information for an informed consent [3]. Here the providers simply act according to what they think will be in the best interests of the patient. These situations frequently happen in hospital emergency rooms and when emergency medical personnel arrive on the scene of an accident or sudden illness.

The emergency exception to informed consent is often quite obvious, but this is not always so. It does not apply, for example, when personnel taking care of somebody in an emergency happen to know what the patient wants. In such a situation, they would not do what they think is the best for the patient but what they know the patient wants.

It is important to note that the emergency exception that allows physicians to do what they think is the best for the patient without obtaining informed consent from the patient or proxy has one major restriction, namely, they cannot do what they think is the best if it is otherwise than what they know the patient or proxy wants. Sometimes, for example, emergency department personnel might know from previous admissions that a particular patient from a local nursing home desires only palliative care. If that patient arrives by ambulance at the same emergency department, it is hard to see how it would be morally reasonable for physicians to take aggressive measures to keep the patient alive when, even though there is no time to obtain consent for orders not to attempt resuscitation or not to intubate, they know he or she or a proxy has decided not to have aggressive life-sustaining measures performed.

Patients accessing emergency care services can present with complaints that are extremely diverse, and the way doctors, nurses and paramedics elicit information from patients predominantly focuses on obtaining biomedical details [4]. In some cases, this approach is warranted, as the urgent need to identify signs and symptoms of life-threatening illness or injury is paramount. Yet, $90 \%$ of patients accessing emergency services are not critically ill or injured but seek help and advice. In addition to seeking advice, patients may also be anxious, frightened, intoxicated, misusing drugs or have unhealthy lifestyles. They may have psychosocial reaction to physical disease or vice versa-physical illness such as irritable bowel syndrome, asthma, tension headache can be triggered by psychosocial factors. The effects and interpretation of illness will trigger a different response to the individual depending on their view and experiences. All these factors will have different needs and concerns and it is important to elicit these concerns within a consultation. However, it has been found that nurses working in emergency care disregard the potential for anxiety and the need for support and reassurance in patients who are not severely ill or injured. In addition, where communication skills of junior doctors working in emergency departments have been researched, they are found to use approaches considered to be more physician/illness orientated than patient-centred. By way of similarities of patient presentations in the pre-hospital setting, this could equally be assumed for paramedic practice. 


\section{Dental Crisis}

Medical emergencies by their very nature can occur at any time, without warning and not necessarily in the clinical environment [5]. It is therefore essential to be able to recognise the nature of an emergency as soon as it occurs and to have the knowledge, prficien cy and corfidence to be able to undertake the appropriate remedial action.

Therapists and hygienists treat patients of all ages and it is inevitable that some of these patients will have significant medical conditions and take medication, both of which may necessitate a modification to dental treatment. In addition, many patients will experience anxiety associated with their treatment. It is to be expected that acute medical conditions will occur in a dental practice, albeit rarely. It is worth remembering that friends or family who often accompany patients, other visitors to the practice and staff may become unwell and require urgent attention. Medical emergencies can therefore occur anywhere on the premises, not just in the surgery. It is essential that all dental healthcare workers should have the knowledge and skills to recognise and provide appropriate immediate medical care for emergencies that might present in dental practice. In some instances, this will require the provision of life-saving measures prior to the arrival of specialist help.

Hygienists and therapists are capable of independent practice and may work when a dentist is away from the dental practice. When the dentist is present, it is probable that he or she will assume the role of team leader in a medical emergency, although another, more experienced clinical member of the team may assume this role. In the event of an emergency, it is hoped that those present would work as a team, with many of the staff making valuable contributions to the management of the patient. However, a dentist may not be on the premises and a therapist/hygienist may be the most senior person and lead the team; in fact, he or she may be the only staff member present. It is therefore important that the hygienist/therapist understands their role fully in a medical crisis and has a clear idea of what actions they would be prepared to carry out.

\section{Dental Emergencies}

Dental professionals continue to owe their patient a duty of care in an emergency situation [6]. They are expected to be able to respond effectively to the common emergencies that might arise in dental surgeries. They are expected to ensure that their practice is up to date and evidence based.

In relation to treatment decisions taken in an emergency, a dental professional will not be found negligent simply because the reasonably competent dentist would have made a different decision, given more time and information.

Moreover, the skill required in the execution of treatment may be somewhat lower because an emergency may overburden the available resources, and, if a dental professional is forced by circumstances to do too many things at once, the fact that he or she does one of them incorrectly would not lightly be taken as negligence.

The extent to which the mouth and its associated structures will need to be examined will depend on the nature of the problem at hand and the degree of urgency it presents [7]. Clearly a detailed head and neck examination will not be required in every patient (e.g., a patient with an obvious mucocele of the lip), but then again, it is important not to jump to conclusions too early and miss something more important. Like skin lesions, many oral conditions are diagnosed to an extent by "pattern recognition" and with experience, accurate "spot diagnoses" become easier. However, for the novice, it is best to start methodically and be thorough. It is important to have a system which ensures that all the hard and soft tissues are examined (including the cervical lymph nodes, jaw bones, and TMJ) and that any lesion that is encountered is also assessed carefully, noting its key features. A mirror, bright light, and tongue depressor are all required.

\section{Pain}

Pain is a highly subjective experience and has been flaed as occurring when and where the patient says it does [8]. Pain is dened by the In ternational Association for the Study of Pain as "an unpleasant sensory and emotional experience associated with actual or potential tissue damage, or described in terms of such damage". It is difficult to determin e a cut-off point for when acute becomes chronic pain but estimates vary from 3 to 12 months.

Chronic pain conditions affecting the oro-facial region can originate from any of the multitude of tissue types present in the head and neck: vascular, nervous, muscular, bony and cartilage, amongst others. Chronic pain conditions must start at a chronological point. They can start either insidiously, or suddenly with an acute limitation of everyday function due to the severity and urgency of the pain experienced. In the latter case, patients may 
present "acutely" to the dental practitioner. The presentation of their complaint may be complicated by the rich sensory oro-facial peripheral innervations. Messages from the peripheral nociceptors can diverge or converge, and therefore, pain may be reported, felt and perceived, to be related to teeth or other oral structures, but may originate from another anatomical site.

Patients with oro-facial pain may report two or more interlinked chief complaints but describe them as a single complaint. If it is felt that this is the case, it is important to discuss this with the patient and clarify whether or not there are two or more definite, separate, issues/diagnoses, or that they are indeed one complaint. This will help to make taking the history of the presenting complaint easier and create less confusion in the clinician and patients' minds. It may also make diagnosis and treatment easier. If there are indeed multiple complaints, this should be made clear in the notes by enumerating the complaints so that anyone reading the notes can quickly reference and relate findings and diagnoses to complaints.

\section{Treatment}

It has been argued that respect for a patient's autonomy requires a dentist to obtain a patient's consent before any dental treatment is carried out [9]. However, some patients will lack the capacity to make autonomous treatment decisions for themselves: for example, young children by virtue of their underdeveloped cognitive skills and instability of life values, adults with cognitive deficits and the unconscious patient in an emergency situation. How should a dentist approach treatment of such patients? Strictly, respect for the patient's autonomy cannot be the guiding ethical principle as these patients are incapable of making autonomous choices. Although non-autonomous, these patients still warrant respect as persons and can be considered to have a right of self-determination which they are incompetent to exercise.

Treatment decisions on behalf of such patients must therefore be taken by surrogate decision makers and consent by a surrogate is called proxy consent. Even in an emergency, reasonable attempts should be made to obtain consent from an appropriate surrogate. The best surrogate decision maker is someone who knows the patient's values and preferences and will usually be a close relative of the patient. Surrogate decision makers can reach decisions on behalf of non-autonomous patients on one of two bases: a substituted judgement or a best interests standard. The former standard attempts to replicate the decision that the patient would have made had he or she been capable. This standard is only appropriate for incompetent adults who were previously competent. For children and adults who have never been competent the best interests standard is appropriate. Thus it will be seen that even in the case of incompetent patients the ethical principle of respect for autonomy prevails (as far as possible) over beneficence for the patient.

\section{Responsibility of the Physicians}

The aim is to provide excellence in emergency department (ED) care by cultivating the following desirable habits [10]:

- $\quad$ Listen to the patient.

- Exclude the differential diagnoses (“rule out”) and refine the possible diagnosis (“rule in”) when assessing any patient, starting with potentially the most life-or limb-threatening conditions, and never trivializing.

- Seek advice and avoid getting out of depth by asking for help.

- Treat all patients with dignity and compassion.

- Make sure the patient and relatives know at all times what is happening and why, and what any apparent waits are for.

- Maintain a collective sense of teamwork, by considering all ED colleagues as equals whether medical, nursing, allied health, administrative or support services.

- Consistently make exemplary ED medical records.

- Communicate whenever possible with the general practitioner (GP).

- Know how to break bad news with empathy.

- $\quad$ Adopt effective risk management techniques.

The duty of care is a physician's obligation to provide treatment according to an accepted standard of care [11]. This obligation usually exists in the context of a physician-patient relationship but can extend beyond it in some circumstances. The physician-patient relationship clearly arises when a patient requests treatment and the physician agrees to provide it. However, creation of this relationship does not necessarily require mutual assent. An unconscious patient presenting to the ED is presumed to request care and the physician assessing such a patient is 
bound by a duty of care. The Emergency Medical Treatment and Active Labor Act (EMTALA) requires ED physicians to assess and stabilize patients coming to the ED before transferring or discharging them. Such an assessment presumably creates the requisite physician-patient relationship.

When caring for a patient, a physician is obligated to provide treatment with the knowledge, skill, and care ordinarily used by reasonably well-qualified physicians practicing in similar circumstances. In some jurisdictions, these similar circumstances include the peculiarities of the locality in which the physician practices. This locality rule was developed to protect the rural practitioner who was sometimes deemed to have less access to the amenities of urban practices or education centers. However, the locality rule is being replaced by a national standard of care in recognition of improved information exchange, ease of transportation, and the more widespread use of sophisticated equipment and technology.

Establishing the standard of care in a given case requires the testimony of medical experts in most circumstances, unless the breach alleged is sufficiently egregious to be self-evident to the lay jury member-for example, amputating the wrong limb or leaving surgical implements in the operative field. A physician specializing in a given field will be held to the standard of other specialists in the same field, rather than to the standard of nonspecialists.

To be eligible to receive federal funds such as Medicare and Medicaid, hospitals with an emergency department must offer emergency and stabilizing treatment services to the public without bias or discrimination [12]. The Emergency Medical Treatment and Active Labor Act is a comprehensive federal law that obligates hospitals offering emergency services to do so without consideration of a patient's ability to pay. It's important to note that this obligation does not apply to inpatients or non-emergent conditions. The absence of bias in the delivery of care should not be misunderstood to suggest all hospitals must provide all medical services, but rather the services they choose to offer must be delivered without bias to the individual patient.

A hospital and its entire staff owe a duty of care to patients admitted for treatment [13]. Following an emergency call, the ambulance service has a duty to respond and provide care. Accident \& Emergency (A\&E) departments have a duty of care to treat anyone who present themselves and are liable for negligence if they send them away untreated. Hospitals without an A\&E facility will display signs stating the location of the nearest A\&E department. This ensures that the hospital could not be held negligent if a patient presented and required emergency treatment as the hospital or its staff had never assumed a duty of care. Once a patient is handed over, a duty of care is created between the patient and the practitioner and this cannot be terminated unless the patient no longer requires the care or the carer is replaced by another equally qualied, competent person. It is therefore extremely important that practitioners are aware of their local policies, professional standards and their scope of practice to avoid becoming liable for litigation by putting a patient at risk, delivering ineffective care or breaching their duty of care.

\section{Conclusion}

Emergency conditions in dentistry are associated with patients who come with symptoms of pain or swelling, which requires immediate diagnosis and treatment. Emergency conditions are a big challenge for any clinician. They require knowing of the symptoms, proper diagnosis and a very quick response to help the patient as soon as possible. Each procedure must be performed in accordance with the rules of the profession in order to minimize the occurrence of any pain. It is important to highlight that each patient has a different perception of pain and a reaction to pain. Because of that, timely and proper reaction is extremely important.

\section{References}

[1] Jones, T. R. (2011). “Approach to the Emergency Department Patient” in Stone, C. K.; Humphries, R. L. (eds): “CURRENT Diagnosis And Treatment Emergency Medicine, Seventh Edition”, The McGraw-Hill Companies, New York, USA, p. 1.

[2] Reilly, M. J., Markenson, D. S. (2011). “Introduction to Hospital and Healthcare Emergency Management” in Reilly, M. J.; Markenson, D. (eds): "Health Care Emergency Management-Principles and Practice”, Jones \& Bartlett Learning, Sudbury, USA, pp. 5-6.

[3] Devettere, R. J. (2010). "Practical Decision Making in Health Care Ethics—Cases and Concepts.” Third Edition, Georgetown University Press, Washington, USA, pp. 83-84.

[4] Gubbins, K., Nixon, V. (2013). “Consultation and Communication Skills” in Nixon, V. (ed): "Professional Practice in Paramedic, Emergency and Urgent Care”, John Wiley \& Sons, Ltd, Chichester, UK, pp. 23.

[5] Longman, L., Balmer, C. (2006). "Medical emergencies and their management” in Ireland, R. (ed): “Clinical Textbook of Dental Hygiene and Therapy”, Blackwell Munksgaard, Blackwell Publishing Company, Oxford, UK, pp. 381-382. 
[6] Griffith, R. (2014). "Professional, ethical and legal issues” in Jevon, P. (ed): "Basic Guide to Medical Emergencies in the Dental Practice”, Second Edition, Wiley Blackwell, John Wiley \& Sons, Ltd, Chichester, UK, pp. 213-214.

[7] Perry, M. (ed.) (2018). “Head, Neck and Dental Emergencies.” Second Edition, Oxford University Press, Oxford, UK, p. 408.

[8] Durham, J. (2012). “Acute Presentations of Chronic Oro-Facial Pain Conditions” in Greenwood, M.; Corbett, I. (eds): “Dental Emergencies”, Wiley Blackwell, John Wiley \& Sons, Ltd, Chichester, UK, pp. 85-86.

[9] Corless-Smith, D. (2002). “Consent to treatment” in Lambden, P. (ed): “Dental Law And Ethics.” Radcliffe Medical Press Ltd, Abingdon, UK, pp. 81.

[10] Brown, A. F. T., Cadogan, M. D. (2011). “Emergency Medicine—Diagnosis and Management.” Sixth Edition, Hodder Arnold, London, UK, p. 446.

[11] Eckerline, jr., Ch. A., Brantley, J. C. (2011). "Legal Aspects of Emergency Care” in Stone, C. K.; Humphries, R. L. (eds): "CURRENT Diagnosis And Treatment Emergency Medicine, Seventh Edition.” The McGraw-Hill Companies, New York, USA, p. 45.

[12] Wollf, A. (2016). “Types of Emergency Departments” in Solheim, J. (ed): “Emergency Nursing—The Profession, The Pathway, The Practice”, Sigma Theta Tau International, Indianapolis, USA, p. 162.

[13] Latcham, K. (2013). "Professional and Legal Issues”, in Nixon, V. (ed): "Professional Practice in Paramedic, Emergency and Urgent Care”, John Wiley \& Sons, Ltd, Chichester, UK, p. 135. 